\title{
CONSECUENCIAS DE LA EXPOSICIÓN INVOLUNTARIA A MATERIAL SEXUAL EN ADOLESCENTES
}

\author{
Beatriz Gil-Juliá, \\ bgil@uji.es \\ Jesús Castro-Calvo, \\ Estefanía Ruiz-Palomino, \\ Marta García-Barba, \\ Rafael Ballester-Arnal \\ Salusex-Unisexsida. \\ Dpto. Psicología Básica, Clínica y Psicobiología. \\ Universitat Jaume I de Castellón (Spain)
}

Fecha de Recepción: 14 Febrero 2018

Fecha de Admisión: 10 Abril 2018

\section{RESUMEN}

Internet se ha convertido en una herramienta útil para nuestros adolescentes pero no está exenta de riesgos, siendo uno de ellos la exposición involuntaria (EI) a material sexual con las consecuencias que dicha exposición puede tener en su desarrollo psicosexual.

Nuestro objetivo fue analizar las consecuencias derivadas de la El a cibersexo teniendo en cuenta posibles diferencias en función de la edad de la primera El, el género y tipo de contenido visto. 284 adolescentes (47,9\% chicos; $52,1 \%$ chicas) de Castellón (Spain), de entre 13-17 años (Media=14,8;DT=0,98) cumplimentaron un cuestionario diseñado ad hoc para la evaluación de diferentes dimensiones implicadas en la El al cibersexo.

Los resultados muestran una frecuencia de El a cibersexo alta $(85,9 \%)$ siendo la edad media de la primera exposición 12,1 años (DT=1,66). Entre las principales consecuencias tras la El destacarían los síntomas de evitación/embotamiento emocional (51,4\%), síntomas sexuales (44,7\%) y aumento de activación fisiológica $(39,4 \%)$. En menor medida, síntomas de reexperimentación del suceso $(29,4 \%)$.

Asimismo, se observan correlaciones significativas y negativas entre la edad de la primera El y algunos síntomas de reexperimentación (p.e. imágenes/pensamientos relacionados con la exposición: rho=-,232; $\mathrm{p}=0,001$ ); embotamiento emocional (p.e. menos capaz de sentir emociones/afectos (rho=-,171; $\mathrm{p}=0,020$ ); aumento de activación (p.e. sobresaltarse fácilmente/exageradamente: rho=0,$195 ; p=0,008$ ); y sexuales (p.e. fantasías/impulsos sexuales hacia objetos/situaciones poco habituales: rho=-0,201; $p=0,006)$. En función del género, aparecen diferencias en la reexperimentación 


\section{CONSECUENCIAS DE LA EXPOSICIÓN INVOLUNTARIA A MATERIAL SEXUAL EN ADOLESCENTES}

de imágenes/pensamientos relacionados con la exposición ( $\left.C h i^{2}=13,89 ; \mathrm{p}=0,000\right)$; evitación/embotamiento (p.e. costarle recordar detalles de la exposición: $\mathrm{Chi}^{2}=5,99 ; \mathrm{p}=0,010$ ); aumento de activación (p.e. dificultades concentración: $C \mathrm{i}^{2}=8,06 ; \mathrm{p}=0,004$ ); y síntomas sexuales (p.e. fantasías/impulsos sexuales hacia objetos/situaciones poco habituales: $\mathrm{Chi}^{2}=12,84 ; \mathrm{p}=0,000$ ), siendo todos más frecuentes en hombres, excepto los síntomas de evitación. Finalmente, en función de haber visto 0 no determinados contenidos se observan diferencias significativas en todos los bloques evaluados.

Es necesario educar sexualmente a nuestros adolescentes para minimizar el impacto negativo de la El a material sexual.

Palabras clave: exposición involuntaria; material sexual; adolescentes; consecuencias; ciberseXO

\section{ABSTRACT}

Consequences of unwanted exposure to online sexual contents in adolescents.

The Internet has become a useful tool for our adolescents but it is not free of risks. One of them is the unwanted exposure (UE) to sexual content with the consequences that such exposure may have on their psychosexual development.

Our objective was to analyse the consequences of being exposed to cybersex taking into account possible differences depending on the age of the first UE, the gender and type of content seen. 284 adolescents (47.9\% boys; $52.1 \%$ girls) from Castellón (Spain), aged 13-17 years (mean=14.8; $\mathrm{SD}=0.98$ ) completed an ad hoc questionnaire for the evaluation of different dimensions involved in the UE to cybersex.

Results show a high frequency of UE to cybersex (85.9\%) being the mean age of the first exposure 12.1 years $(S D=1.66)$. Among the main consequences after the $U E$, we can highlight symptoms of avoidance/emotional dullness (51.4\%), sexual symptoms (44.7\%) and increased physiological activation (39.4\%). To a lesser extent, symptoms of reexperimentation $(29.4 \%)$ appeared.

Likewise, significant and negative correlations are observed between the age of the first UE and some symptoms of reexperimentation (eg., images/thoughts related to exposure: rho=$.232 ; p=0.001$ ); emotional dullness (eg., less ability to feel emotions/affects (rho=-.171; $p=0.020$ ); increased activation (eg., startling easily/exaggeratedly: rho $=-0.195 ; \mathrm{p}=0.008$ ); and sexual symptoms (eg., fantasies/impulses to unusual objects/situations: rho=-0.201; $p=0.006$ ). Depending on the gender, there are differences in the reexperience of images/thoughts related to exposure $\left(\mathrm{Chi}^{2}=13.89 ; \mathrm{p}=0.000\right)$; avoidance/emotional dullness (eg., difficulty in remembering details of the exposure: $\quad C h i^{2}=5.99 ; p=0.010$ ); increased activation (eg., concentration difficulties: $\mathrm{Chi}^{2}=8.06 ; \mathrm{p}=0.004$ ); and sexual symptoms (eg., sexual fantasies/impulses towards unusual objects/situations: $\mathrm{Chi}^{2}=12.84 ; \mathrm{p}=0.000$ ). All of them are more frequent in men, except for the avoidance symptoms. Finally, significant differences are observed in all the symptoms blocks evaluated based on having or not having seen certain contents.

It is necessary to educate our adolescents sexually to minimize the negative impact of UE to online sexual content.

Keywords: unwanted exposure; sexual material; teenagers; consequences; cybersex

Este trabajo ha sido financiado por el proyecto P1.1B2015-82 (Universitat Jaume I)

\section{ANTECEDENTES}

El uso de internet se ha generalizado de tal manera que la mayoría de hogares dispone de esta herramienta habiéndose convertido en una necesidad casi básica dadas las múltiples ventajas que 
nos aporta. Datos de la última encuesta del Instituto Nacional de Estadística sobre equipamiento y uso de las nuevas tecnologías de la información y la comunicación (TIC) en hogares españoles (INE, 2017) revelan que el $83,4 \%$ de los hogares presentan conexión a internet y que el $82,7 \%$ disponen de conexión de banda ancha. Asimismo, el 84,6\% de los usuarios (de entre 16 y 74 años) se ha conectado a internet durante los últimos tres meses, porcentaje que aumenta al 95,1\% cuando exploramos el uso de internet entre los menores de la casa (niños de entre 10 y 15 años). De hecho, algunos estudios asocian el incremento del uso de TIC a la demanda efectuada por parte de los menores, es decir, habrá un mayor uso de nuevas tecnologías y con ellas de internet en aquellos hogares en los que residen niños menores de edad (Observatorio de la Infancia en Andalucía, 2010). En todos los países europeos, incluido España, se registra un mayor porcentaje de acceso a Internet en los hogares con hijos/as a cargo (Eurostat, 2015).

Internet se ha convertido por tanto en una herramienta prácticamente imprescindible para niños y adolescentes proporcionándoles gran riqueza de información y oportunidades. Sin embargo, con el uso de internet se exponen también a importantes peligros como ser víctimas de solicitudes sexuales no deseadas, acoso o exposición involuntaria (EI) a material sexual (Livingstone, Haddon, Görzig \& Ólafsson, 2011), entendida esta última como "la exposición a imágenes de personas desnudas o manteniendo relaciones mientras una persona realiza una búsqueda online, navega por la red 0 abre su correo electrónico, siempre y cuando no busque voluntariamente ni espere ver material sexual" (p.337) (Mitchell, Finkelhor \& Wollak, 2003). Esta definición de El es ampliada por Bryant (2009) unos años más tarde al considerar como El aquellas situaciones de exposición que tienen lugar durante la interacción con otros usuarios. De modo que globalmente se considerarían como El todas aquellas situaciones en las que una persona pueda recibir solicitudes sexuales no deseadas, sea acosada sexualmente a través de Internet 0 sea expuesta a contenidos sexuales explícitos de forma inesperada (Wollack, Mitchell \& Finkelhor, 2007).

Diferentes investigaciones han intentado cuantificar la magnitud de la El a material sexual online ofreciendo datos de prevalencia que oscilan entre $23 \%$ y $44 \%$ en edades comprendidas entre los 13-17 años (Ballester-Arnal, Giménez-García, Gil-Llario \& Castro-Calvo, 2016; Jones, Mitchell \& Finkelhor, 2012; Mitchell et al., 2003; Mitchell, Wolak \& Finkelhor, 2007) suscitando gran preocupación en torno a la capacidad que estos niños y adolescentes puedan tener para manejar de forma adecuada esta información (Delmonico \& Griffin, 2008). Todo ello cobra especial relevancia si tenemos en cuenta que dicha exposición puede constituir una experiencia negativa para los adolescentes (Ševíková, Simon, Daneback \& Kvapilík, 2016) e incluso tener efectos negativos en su desarroIlo psicosexual (Peter \& Valkenburg, 2008). Aunque existe una cierta controversia y existen todavía pocos estudios al respecto de los posibles efectos beneficiosos o perjudiciales del cibersexo en sus usuarios, algunos autores refieren que a largo plazo, la El podría fomentar en los menores, entre otros, valores y creencias sexuales erróneas, actitudes sexuales excesivamente permisivas, preocupaciones sexuales, promiscuidad sexual, edad de inicio en las relaciones sexuales excesivamente temprana, reacciones emocionales intensas (ansiedad, depresión, síntomas de TEPT) 0 un pobre autoconcepto (Owens, Behun, Manning \& Reid, 2012).

El impacto que la El puede tener a nivel psicológico y también en el desarrollo sexual de nuestros niños y adolescentes contrasta con la escasez de estudios realizados sobre todo en nuestro contexto. Por ello el objetivo del presente trabajo fue analizar las consecuencias derivadas de la El al cibersexo teniendo en cuenta posibles diferencias en función de la edad de la primera El, el género y el tipo de contenido visto. 


\section{CONSECUENCIAS DE LA EXPOSICIÓN INVOLUNTARIA A MATERIAL SEXUAL EN ADOLESCENTES}

\section{MÉTODO}

\section{Participantes}

En este estudio han participado 284 adolescentes (47,9\% chicos; $52,1 \%$ chicas) de 3 institutos de Castellón de la Plana (España) con edades comprendidas entre los 13 y los 17 años (Media=14,8; $\mathrm{DT}=0,98)$. Los participantes del estudio estaban cursando 3ํ de la ESO (55\%) y $4^{0}$ de la ESO $(45 \%)$ en el momento de la evaluación.

\section{Instrumentos}

Para la evaluación de los participantes se ha utilizado un Cuestionario Ad-Hoc para explorar distintos aspectos de la El. Este instrumento está compuesto por 12 ítems con múltiples formatos de respuesta (escala tipo likert, respuesta dicotómica, elección múltiple, etc.) que nos permiten explorar distintas dimensiones de la El desde la frecuencia de exposición o la edad de la primera exposición hasta las reacciones a la El y sus consecuencias. Para este trabajo se seleccionaron los ítems que medían frecuencia de El, edad de la primera El, tipo de contenido visto en la El y consecuencias tras la exposición involuntaria a material sexual.

\section{Procedimiento}

El instrumento de evaluación se aplicó en el marco de una investigación más amplia en el contexto escolar. Un psicólogo especialista en prevención fue el encargado de administrar los cuestionarios en clases de 3ํy 4ํㅡㄹ la ESO de 3 institutos de Castellón de la Plana. La aplicación del cuestionario (en formato lápiz y papel) fue grupal, si bien el encargado de la aplicación se aseguró de mantener el rigor exigido en toda investigación separando debidamente a los alumnos con el fin de mantener el anonimato y confidencialidad de las respuestas, así como solucionando cualquier duda que pudiera surgir.

\section{Análisis de los datos}

Los datos se analizaron mediante el paquete estadístico SPSS Versión 23.0. Para el cálculo de la prevalencia de la El se utilizó el total de participantes que componen la muestra. Sin embargo, para el resto de análisis se seleccionaron únicamente aquellos participantes que afirmaron haber sido expuestos de forma involuntaria al menos en alguna ocasión a material sexual online. De esta forma nos aseguramos la representatividad y posible generalización de los resultados obtenidos. Para el análisis de asociaciones entre variables se llevaron a cabo correlaciones de Spearman y para Ios análisis diferenciales la prueba estadística $\mathrm{Chi}^{2}$ por tratarse de variables categóricas.

\section{RESULTADOS}

\section{Frecuencia de exposición involuntaria}

Los resultados muestran una frecuencia de El a cibersexo alta (85,9\%). La mayoría de los adolescentes evaluados afirmó haber sido expuesto involuntariamente a material sexual online al menos en alguna ocasión siendo la edad media de la primera exposición 12,1 años (DT=1,66). De entre los adolescentes que afirmaron haber sido expuestos los mayores porcentajes están representados por las opciones "casi nunca" (43,9\%) y "algunas veces" (35,7\%). En la figura 1 podemos observar que los porcentajes de exposición involuntaria tienden a disminuir en las opciones de respuesta que implican mayor exposición. No obstante, un porcentaje nada despreciable de los adolescentes dice haber sido expuesto involuntariamente a este tipo de contenido en internet muchas veces $(4,1 \%) 0$ muchísimas veces $(2,5 \%)$. 
Figura 1. Frecuencia de exposición involuntaria en función del género

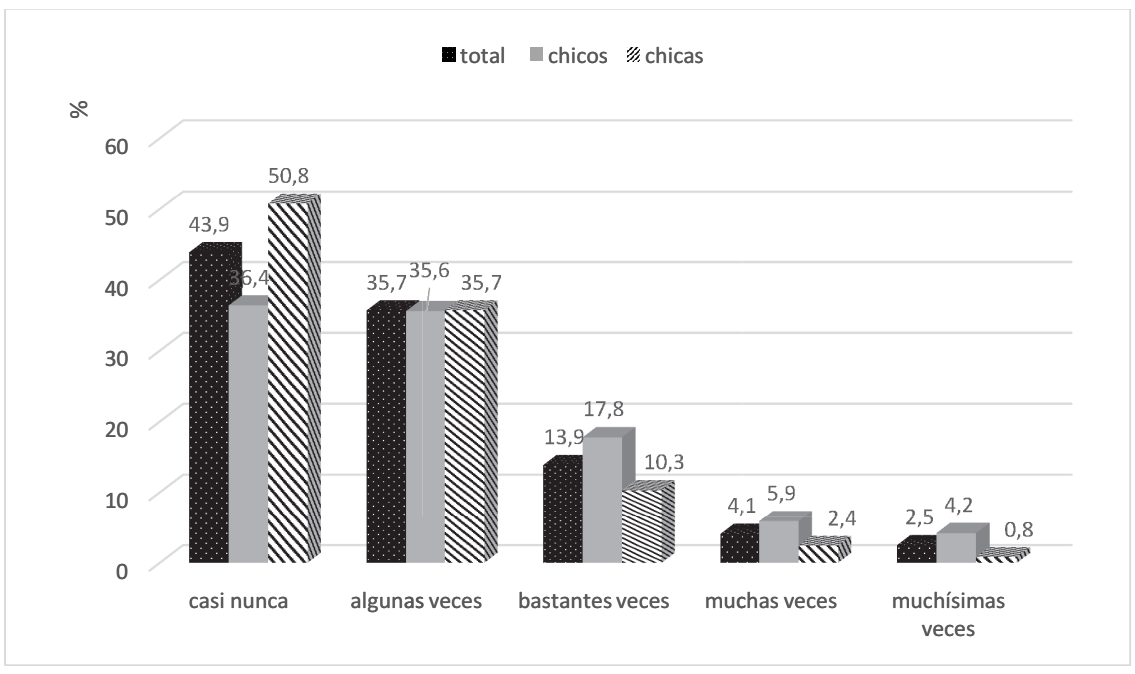

En función del género, el porcentaje de chicos que afirmó haber sido expuesto (90,1\%) supera ligeramente al porcentaje de chicas (82,4\%), no alcanzando estas diferencias la significación estadística (Chi-cuadrado=3,48; $p=0,062$ ). En cuanto a la edad media de la primera exposición, los chicos fueron expuestos significativamente antes que las chicas $(t=-2,517 ; p=0,013)$. Asimismo, en la frecuencia de El observamos que los porcentajes asignados a cada una de las opciones de respuesta difieren significativamente en función del género (Chi-cuadrado=10,12; $p=0,038$ ).

\section{Tipo de contenido}

Durante la El las escenas más vistas por los adolescentes fueron "gente desnuda que no está teniendo relaciones sexuales" (61,2\%), "relación sexual entre un hombre y una mujer" $(54,1 \%)$, "personas desnudas enseñando los genitales" (48,3\%) y "dibujos manga de contenido sexual" $(36,8 \%)$. Si bien otros contenidos han sido vistos en menor medida es importante tener en consideración aquellos porcentajes que hacen referencia a "juegos sexuales que implican sometimiento" (14,9\%), "actividad sexual con adolescentes" (7,9\%), "violación o violencia sexual" (2,5\%) y "actividad sexual con niños pequeños" $(1,7 \%)$.

Si analizamos el tipo de contenido visto durante la El en función del género observamos diferencias significativas en las escenas que incluyen "gente desnuda que no está teniendo relaciones sexuales" (Chi-cuadrado=6,36; $p=0,012$ ), "relación sexual entre un hombre y una mujer" (Chi-cuadrado=18,51; $p=0,000$ ) y "relación sexual entre personas y animales" (Chi-cuadrado=4,49; $p=0,034$ ) habiendo sido estas dos últimas escenas más vistas por chicos (véase tabla 1). 


\section{CONSECUENCIAS DE LA EXPOSICIÓN INVOLUNTARIA A MATERIAL SEXUAL EN ADOLESCENTES}

Tabla 1. Tipo de contenido sexual visto en la El

\begin{tabular}{lcccc}
\hline Escenas vistas & Total & Chicos & Chicas & Chi' $^{2}$ \\
\hline $\begin{array}{l}\text { Gente desnuda que no está teniendo relaciones } \\
\text { sexuales }\end{array}$ & $61,2 \%$ & $53 \%$ & $68,8 \%$ & $\mathbf{6 , 3 6 *}$ \\
Personas desnudas enseñando los genitales & $48,3 \%$ & $43,6 \%$ & $52,8 \%$ & 2,05 \\
Relación sexual entre un hombre y una mujer & $54,1 \%$ & $68,4 \%$ & $40,8 \%$ & $\mathbf{1 8 , 5 1 * * *}$ \\
Relaciones sexuales entre personas del mismo sexo & $18,2 \%$ & $20,5 \%$ & $16 \%$ & 0,83 \\
Relaciones sexuales entre más de dos personas & $24,4 \%$ & $27,4 \%$ & $21,6 \%$ & 1,08 \\
Uso de heces y orina durante las relaciones sexuales & $4,1 \%$ & $5,1 \%$ & $3,2 \%$ & 0,57 \\
Juegos sexuales que implican sometimiento & $14,9 \%$ & $13,7 \%$ & $16 \%$ & 0,26 \\
Actividad sexual entre personas y animales & $5,4 \%$ & $8,5 \%$ & $2,4 \%$ & $\mathbf{4 , 4 9 *}$ \\
Dibujos manga de contenido sexual & $36,8 \%$ & $36,8 \%$ & $36,8 \%$ & 0 \\
Violación o violencia sexual & $2,5 \%$ & $1,7 \%$ & $3,2 \%$ & 0,55 \\
Actividad sexual con adolescentes & $7,9 \%$ & $7,7 \%$ & $8 \%$ & 0,01 \\
Actividad sexual con niños pequeños & $1,7 \%$ & $1,7 \%$ & $1,6 \%$ & 0,004 \\
\hline
\end{tabular}

${ }^{*} p<0,05 ; * * p<0,01 ; * * * p<0,001$

\section{Consecuencias tras la El}

Como podemos apreciar en la tabla 2 prácticamente todos los síntomas explorados tras la El parecen estar presentes entre los adolescentes evaluados. De entre estas consecuencias destacarían los síntomas de evitación/embotamiento emocional. Concretamente, al 31,1\% de los adolescentes les cuesta recordar ciertos detalles de la exposición y el 17,8\% evita cualquier cosa que le recuerde a la exposición. Otros síntomas también frecuentes han sido los síntomas sexuales. En este sentido destacan la presencia de fantasías/impulsos sexuales intensos hacia objetos o situaciones poco habituales $(19,9 \%)$ y sentir asco por todo lo relacionado con el sexo $(9,5 \%)$. A estos síntomas les siguen de cerca aquellos relacionados con el aumento de activación fisiológica, por ejemplo, el $12,4 \%$ de los participantes dicen sentirse en alerta tras la exposición y el 9,5\% sobresaltarse con facilidad y de forma exagerada. En menor medida estaría representado el grupo de síntomas de reexperimentación si bien cabe destacar que el 11,2\% de los adolescentes ha tenido imágenes y pensamientos que no podía controlar relacionados con la exposición.

Analizando estas consecuencias en función del género observamos diferencias significativas en la reexperimentación de imágenes/pensamientos relacionados con la exposición siendo esta sintomatología mayor en los chicos $\left(\mathrm{Chi}^{2}=13,89 ; \mathrm{p}=0,000\right)$. Asimismo, los chicos han presentado significativamente más problemas de concentración que las chicas $\left(\mathrm{Chi}^{2}=8,06 ; p=0,005\right)$ y más fantasías/impulsos sexuales intensos hacia objetos 0 situaciones poco habituales $\left(\mathrm{Chi}^{2}=12,84 ; p=0,000\right)$. En cambio, han sido las chicas quienes han presentado más dificultad para recordar ciertos detalles de la exposición $\left(\mathrm{Chi}^{2}=5,99 ; \mathrm{p}=0,014\right)$ (véase tabla 2).

Profundizando en el estudio de las consecuencias experimentadas por los adolescentes tras la El se observan correlaciones significativas y negativas entre la edad de la primera El y algunos síntomas de cada uno de los bloques de consecuencias considerados (véase tabla 2). Dentro de los síntomas de reexperimentación, se han obtenido correlaciones significativas con "tener imágenes/pensamientos relacionados con la exposición" (rho=-0,232; p=0,001) y "tener la sensación de 
estar volviendo a vivir la exposición a ese contenido" (rho=-0,211; $p=0,004)$. También se han obtenido relaciones significativas con síntomas de embotamiento emocional, concretamente con el hecho de "ser menos capaz de sentir emociones/afectos" (rho=-0,171; $p=0,020$ ) y con "sentir menos interés por cosas que solía hacer" (rho=-0,199; $p=0,007$ ). Dentro de los síntomas relacionados con el aumento de activación fisiológica se han encontrado correlaciones significativas con tener "dificultades para conciliar/mantener el sueño" (rho=-0,157; $p=0,032$ ), "problemas de concentración" (rho=-0,266; $p=0,000)$ y "sobresaltarse fácilmente/exageradamente" (rho=-0,195; $p=0,008$ ). Finalmente, los resultados también han mostrado correlaciones significativas entre la edad de la primera exposición y síntomas sexuales como tener "fantasías/impulsos sexuales hacia objetos/situaciones poco habituales ( $r h 0=-0,201 ; p=0,006$ ). La presencia de todos estos síntomas ha sido mayor cuanto más pronto fueron expuestos los adolescentes de forma involuntaria a contenido sexual online.

Tabla 2. Consecuencias de la El en función del género y edad de la primera EI

\begin{tabular}{|c|c|c|c|c|c|}
\hline & & \multicolumn{3}{|c|}{ Género } & \multirow{2}{*}{$\frac{\text { Edad 1a }^{\text {a }} \text { El }}{\text { Rho }}$} \\
\hline & Total & Chicos & Chicas & Chi2 & \\
\hline \multicolumn{6}{|l|}{ Síntomas de reexperimentación del suceso } \\
\hline $\begin{array}{l}\text { - Imágenes y pensamientos que no puedo } \\
\text { controlar relacionados con la exposición }\end{array}$ & $11,2 \%$ & $19,1 \%$ & $4 \%$ & $13,89 * * *$ & $-0,232 * *$ \\
\hline $\begin{array}{l}\text { - Sensación de volver a vivir la exposición a ese } \\
\text { contenido }\end{array}$ & $6,6 \%$ & $7,8 \%$ & $5,6 \%$ & 0,50 & $-0,211 * *$ \\
\hline $\begin{array}{l}\text { - Me siento mal cada vez que recuerdo el } \\
\text { contenido de la exposición }\end{array}$ & $8,3 \%$ & $9,6 \%$ & $7,1 \%$ & 0,46 & 0,050 \\
\hline $\begin{array}{l}\text { - Síntomas fisiológicos cuando algo me } \\
\text { recuerda el contenido }\end{array}$ & $3,3 \%$ & $5,2 \%$ & $1,6 \%$ & 2,47 & $-0,062$ \\
\hline - Lo anterior me ocurre de forma frecuente & $3,7 \%$ & $5,2 \%$ & $2,4 \%$ & 1,35 & $-0,035$ \\
\hline \multicolumn{6}{|l|}{ Síntomas de evitación o embotamiento } \\
\hline $\begin{array}{l}\text { - Evito cualquier cosa que me recuerda a la } \\
\text { exposición }\end{array}$ & $17,8 \%$ & $13 \%$ & $22,2 \%$ & 3,46 & 0,005 \\
\hline $\begin{array}{l}\text { - Me cuesta recordar ciertos detalles de la } \\
\text { exposición }\end{array}$ & $31,1 \%$ & $23,5 \%$ & $38,1 \%$ & $5,99 *$ & 0,072 \\
\hline - Siento menos interés por cosas que solía hacer & $3,3 \%$ & $5,2 \%$ & $1,6 \%$ & 2,47 & $-0,199 * *$ \\
\hline $\begin{array}{l}\text { - Empiezo a sentir que los demás no me } \\
\text { importan }\end{array}$ & $0,4 \%$ & $0 \%$ & $0,8 \%$ & 0,92 & $-0,070$ \\
\hline $\begin{array}{l}\text { - Soy menos capaz de sentir emociones o } \\
\text { afectos }\end{array}$ & $2,5 \%$ & $2,6 \%$ & $2,4 \%$ & 0,01 & $-0,171 *$ \\
\hline - No espero nada de mi vida & $1,2 \%$ & $2,6 \%$ & $0 \%$ & 3,33 & $-0,070$ \\
\hline \multicolumn{6}{|l|}{ Síntomas de aumento de la activación } \\
\hline -Dificultades para conciliar/mantener el sueño & $4,6 \%$ & $6,1 \%$ & $3,2 \%$ & 1,17 & $-0,157 *$ \\
\hline - Me siento irritado & $5 \%$ & $6,1 \%$ & $4 \%$ & 0,57 & 0,104 \\
\hline - Problemas de concentración & $7,9 \%$ & $13 \%$ & $3,2 \%$ & $8,06 * *$ & $-0,266 * *$ \\
\hline - Me siento en alerta & $12,4 \%$ & $11,3 \%$ & $13,5 \%$ & 0,26 & 0,101 \\
\hline -Me sobresalto con facilidad y exageradamente & $9,5 \%$ & $9,6 \%$ & $9,5 \%$ & 0 & $-0,195 * *$ \\
\hline \multicolumn{6}{|l|}{ Síntomas sexuales } \\
\hline $\begin{array}{l}\text { - He tenido fantasías/impulsos sexuales intensos } \\
\text { hacia objetos o situaciones poco habituales }\end{array}$ & $19,9 \%$ & $29,6 \%$ & $11,1 \%$ & $12,84 * * *$ & $-0,201 * *$ \\
\hline $\begin{array}{l}\text { - He experimentado menos deseo por mantener } \\
\text { relaciones sexuales o masturbarme }\end{array}$ & $6,6 \%$ & $5,2 \%$ & $7,9 \%$ & 0,72 & 0,030 \\
\hline $\begin{array}{l}\text { - He sentido asco por todo lo relacionado con el } \\
\text { sexo }\end{array}$ & $9,5 \%$ & $6,1 \%$ & $12,7 \%$ & 3,04 & $-0,053$ \\
\hline - He sido incapaz de excitarme sexualmente & $3,3 \%$ & $4,3 \%$ & $2,4 \%$ & 0,72 & 0,080 \\
\hline $\begin{array}{l}\text { - He tenido dudas sobre si me gustan los chicos } \\
\text { o las chicas }\end{array}$ & $5,4 \%$ & $4,3 \%$ & $6,3 \%$ & 0,47 & $-0,056$ \\
\hline
\end{tabular}


Finalmente, en función de haber visto o no determinados contenidos durante la El los resultados muestran diferencias significativas en todos los bloques evaluados (véase tabla 3 ). Concretamente, dentro de los síntomas de reexperimentación observamos mayor presencia de "imágenes/pensamientos que no pueden controlar relacionados con la exposición" en aquellos adolescentes que han sido expuestos a escenas en las que aparecían "relaciones sexuales entre un hombre y una mujer" (Chi-cuadrado $=11,93 ; p=0,001$ ), "relaciones sexuales entre personas del mismo sexo" (Chi-cuadrado=4,86; $p=0,028$ ) y "actividad sexual entre personas y animales" (Chicuadrado=16,67; $p=0,000)$. También tienen más "sensación de estar volviendo a vivir la exposición" Ios que han visto "actividad sexual entre personas y animales" (Chi-cuadrado $=5,91 ; p=0,015$ ) y "actividad sexual con adolescentes" (Chi-cuadrado=6,81; $p=0,009$ ). Asimismo, los adolescentes que han sido expuestos a escenas de "actividad sexual con niños pequeños" en mayor medida "se sienten mal cada vez que recuerdan el contenido de la exposición" (Chi-cuadrado=9,19; $p=0,002$ ). De igual forma ante este último contenido (Chi-cuadrado=5,06; $p=0,024$ ) y ante "relaciones sexuales entre personas del mismo sexo" (Chi-cuadrado=4,43; $p=0,035$ ) los adolescentes presentan más síntomas de reexperimentación de forma frecuente.

Atendiendo a las diferencias encontradas en los síntomas de evitación/embotamiento en función del contenido visto, los resultados señalan más "evitación de cualquier cosa que recuerde a la exposición" en los adolescentes que han sido expuestos a "juegos sexuales que implican sometimiento" (Chi-cuadrado=7,27; $p=0,007$ ) y "actividad sexual con adolescentes" (Chi-cuadrado=5,29; $p=0,021$ ). Asimismo, aquellos adolescentes que han sido expuestos a "juegos sexuales que implican sometimiento" (Chi-cuadrado=7,89; $p=0,005$ ), "actividad sexual entre personas y animales" (Chi-cuadra$d 0=6,16 ; p=0,013$ ) y "actividad sexual con niños pequeños" (Chi-cuadrado=27,37; $p=0,000$ ) "sienten menos interés por cosas que solían hacer". Observamos también mayor incapacidad para sentir emociones y afectos cuando el contenido de la exposición ha sido "juegos sexuales que implican sometimiento" (Chi-cuadrado=12,81; $p=0,000$ ), "violación 0 violencia sexual" (Chi-cuadrado=5,04; $p=0,025)$, "actividad sexual con adolescentes" (Chi-cuadrado $=5,42 ; p=0,020$ ) y "actividad sexual con niños pequeños" (Chi-cuadrado=8,41; $p=0,004$ ).

Por lo que respecta a los síntomas relacionados con el aumento de activación fisiológica, observamos más dificultades para conciliar o mantener el sueño en los adolescentes que han visto "juegos sexuales que implican sometimiento" (Chi-cuadrado=9,96; $p=0,002$ ) y "actividad sexual con niños pequeños" (Chi-cuadrado=21,30; $\mathrm{p}=0,000$ ). Igualmente, los resultados muestran más "problemas de concentración" en aquellos que han sido expuestos al visionado de "relaciones sexuales entre un hombre y una mujer" (Chi-cuadrado $=7,59 ; p=0,006$ ) y "estar más en alerta" en el caso de haber sido expuestos a "actividad sexual con adolescentes" (Chi-cuadrado=6,81; $p=0,009$ ).

Dentro del último bloque de síntomas evaluado, el correspondiente a síntomas sexuales, encontramos también diferencias en función del tipo de contenido. En este sentido, se observan más "fantasías/impulsos sexuales intensos hacia objetos o situaciones poco habituales" en los adolescentes expuestos involuntariamente a escenas de "relación sexual entre un hombre y una mujer" (Chi-cuadrado=20,84; $p=0,000$ ), "relaciones sexuales entre personas del mismo sexo" (Chi-cuadrado=5,08; $p=0,024$ ), "violación 0 violencia sexual" (Chi-cuadrado=8,32; $p=0,004$ ) y "actividad sexual con adolescentes" (Chi-cuadrado=6,24; $p=0,013$ ). Asimismo, los adolescentes expuestos a "actividad sexual con niños pequeños" también han sentido más "asco por todo lo relacionado con el sexo" (Chi-cuadrado=19,99; $p=0,000$ ) y se han sentido más "incapaces de excitarse sexualmente" (Chicuadrado=5,89; $p=0,015)$. Finalmente, entre los que han visto "juegos sexuales que implican sometimiento" también se han observado más "dudas sobre si les gustan los chicos o las chicas" (Chicuadrado=6,99; $p=0,008$ ). Cabe señalar que en algunos casos las diferencias encontradas muestran 
mayor prevalencia de síntomas en aquellos adolescentes que no han sido expuestos a determinados contenidos. Concretamente, los que no han visto "relaciones sexuales entre un hombre y una mujer" y "gente desnuda sin tener relaciones sexuales" han presentado más síntomas relacionados con la "dificultad para recordar detalles de la exposición" (Chi-cuadrado=12,18; $p=0,000$ ) y "sobresaltarse con facilidad" (Chi-cuadrado=5,38; $p=0,020$ ), respectivamente.

Si bien los resultados han mostrado diferencias en todos los bloques de síntomas, también es interesante ver respecto a qué contenidos se observan más efectos en los adolescentes y de qué tipo son estos efectos (véase tabla 3). En este sentido, y por orden decreciente, las escenas que más síntomas han producido han sido ver actividad sexual con niños pequeños (diferencias en todos los bloques de síntomas), actividad sexual con adolescentes (diferencias en todos los bloques de síntomas) y juegos sexuales que implican sometimiento (diferencias en tres bloques de síntomas: evitación/embotamiento, aumento de activación y sexuales), estando igualados estos dos últimos en número de síntomas producidos. En menor medida estarían las escenas que incluyen relaciones sexuales entre un hombre y una mujer (diferencias en todos los bloques de síntomas), relaciones sexuales entre personas del mismo sexo (diferencias en dos bloques de síntomas: reexperimentación y sexuales) y actividad sexual entre personas y animales (diferencias en dos bloques de síntomas: reexperimentación y evitación/embotamiento), también igualados estos dos últimos en número de efectos. Por último, la escena que menos efectos produce es la de ver violencia sexual (diferencias en dos bloques de síntomas: evitación/embotamiento y sexuales). Asimismo, cabe destacar que ver determinados contenidos no produce efectos significativos en los adolescentes. Tal es el caso de las escenas que incluyen personas enseñando genitales, relaciones entre más de dos personas, uso de heces y orina, y dibujos manga de origen sexual.

Tabla 3. Consecuencias de la El en función del tipo de contenido visto

\begin{tabular}{|c|c|c|c|c|c|c|c|c|c|c|c|c|}
\hline & Ítem 1 & Ítem 2 & Ítem 3 & Ítem 4 & Ítem 5 & Ítem 6 & Ítem 7 & Ítem 8 & Ítem 9 & $\begin{array}{c}\text { Ítem } \\
10\end{array}$ & $\begin{array}{c}\text { Ítem } \\
11 \\
\end{array}$ & Ítem 12 \\
\hline $\begin{array}{l}\text { Síntomas de reexperimentación del } \\
\text { suceso }\end{array}$ & ${\mathrm{C} \mathrm{Chi}^{2}}^{2}$ & $\mathrm{CChi}^{2}$ & 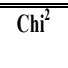 & $\overline{C \mathrm{Chi}^{2}}$ & 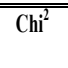 & 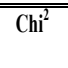 & $\mathrm{Chi}^{2}$ & 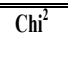 & $\mathrm{Chi}^{2}$ & $\mathrm{CHhi}^{2}$ & $\mathrm{C}_{\mathrm{Chi}}{ }^{2}$ & $\overline{C \mathrm{Chi}^{2}}$ \\
\hline $\begin{array}{l}\text { - Imágenes y pensamientos que no } \\
\text { puedo controlar relacionados con la } \\
\text { exposición }\end{array}$ & 3,74 & $0,0,17$ & $\begin{array}{c}11,93^{*} \\
*\end{array}$ & $4,86^{*}$ & 2,70 & 0,79 & 0,00 & $\begin{array}{c}16,67^{*} \\
\div *\end{array}$ & 1,55 & 0,78 & 0,75 & 0,76 \\
\hline $\begin{array}{l}\text { - Sensación de volver a vivir la } \\
\text { exposición a ese contenido }\end{array}$ & 0,38 & 0,02 & 0,50 & 0,00 & 0,45 & 2,96 & 1,32 & $5,91^{*}$ & 1,19 & 0,98 & $6,81^{\text {*** }}$ & 2,18 \\
\hline $\begin{array}{l}\text { - Me siento mal cada vez que recuerdo el } \\
\text { contenido de la exposición }\end{array}$ & 2,51 & 0,08 & 0,01 & 0,94 & 0,01 & 0,04 & 1,68 & 0,01 & 0,05 & 0,56 & 1,48 & $9,19^{* *}$ \\
\hline $\begin{array}{l}\text { - Síntomas fisiológicos cuando algo me } \\
\text { recuerda el contenido }\end{array}$ & 2,01 & 0,37 & 1,47 & 0,28 & 0,00 & 1,43 & 0,64 & 0,80 & 2,17 & 0,21 & 0,23 & 0,14 \\
\hline $\begin{array}{l}\text { - Lo anterior me ocurre de forma } \\
\text { frecuente }\end{array}$ & 1,15 & 0,82 & 0,61 & $4,43^{*}$ & 0,88 & 0,41 & 1,66 & 0,54 & 0,21 & 0,24 & 0,13 & $5,06^{*}$ \\
\hline \multicolumn{13}{|l|}{ Síntomas de evitación o embotamiento } \\
\hline $\begin{array}{l}\text { - Evito cualquier cosa que me recuerda a } \\
\text { la exposición }\end{array}$ & 0,98 & $\overline{0,07}$ & 0,21 & 2,32 & 2,28 & 0,04 & $7,27^{7 *}$ & 0,93 & 0,02 & 0,00 & 5,29 * & 0,15 \\
\hline $\begin{array}{l}\text { - Me cuesta recordar ciertos detalles de } \\
\text { la exposición }\end{array}$ & 2,83 & 2,88 & $\begin{array}{c}12,18^{*} \\
* *\end{array}$ & 0,29 & 1,08 & 0,00 & 0,07 & 0,00 & 2,14 & 0,62 & 0,29 & 0,08 \\
\hline $\begin{array}{l}\text { - Siento menos interés por cosas que } \\
\text { solia hacer }\end{array}$ & 2,01 & 0,01 & 1,47 & 0,28 & 0,00 & 0,36 & $7,89 \% *$ & $6,16^{*}$ & 0,58 & 0,21 & 0,71 & $\begin{array}{c}27,37 * * \\
*\end{array}$ \\
\hline $\begin{array}{l}\text { - Empiezo a sentir que los demás no me } \\
\text { importan }\end{array}$ & 1,60 & 0,93 & 1,18 & 0,22 & 0,32 & 0,04 & 0,18 & 0,06 & 0,59 & 0,03 & 0,09 & 0,02 \\
\hline $\begin{array}{l}\text { - Soy menos capaz de sentir emociones } 0 \\
\text { afectos }\end{array}$ & 2,06 & 0,54 & 2,13 & 0,98 & 2,22 & 0,27 & $\begin{array}{c}12,81^{*} \\
* *\end{array}$ & 1,51 & 1,11 & $5,04^{*}$ & $5,42^{*}$ & $8,41^{* *}$ \\
\hline - No espero nada de mi vida & 0,03 & 0,27 & 0,52 & 0,67 & 0,97 & 0,13 & 0,54 & 0,17 & 1,13 & 0,08 & 2,67 & 0,05 \\
\hline
\end{tabular}




\section{CONSECUENCIAS DE LA EXPOSICIÓN INVOLUNTARIA A MATERIAL SEXUAL EN ADOLESCENTES}

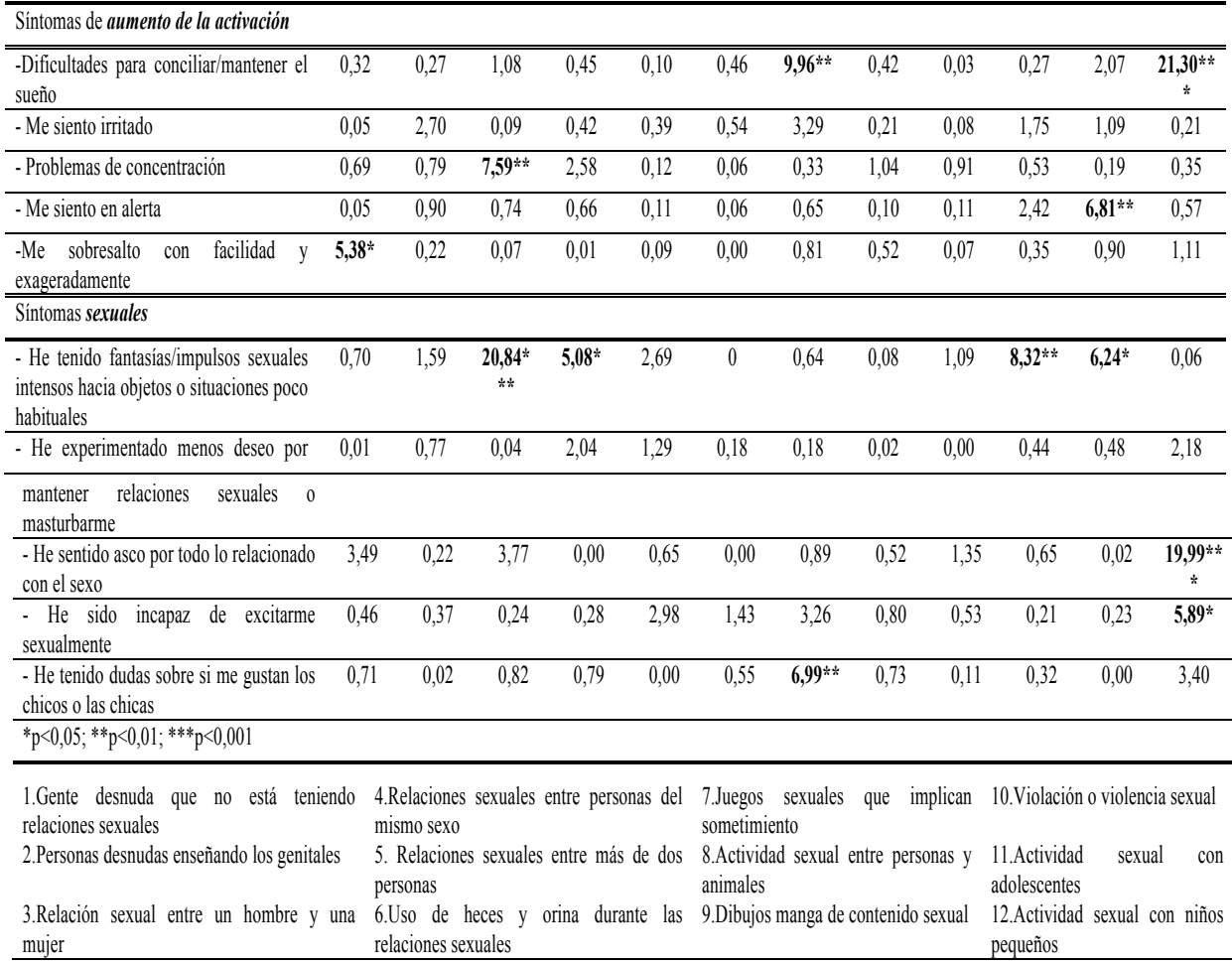

\section{DISCUSIÓN Y CONCLUSIONES}

Los resultados de este trabajo nos permiten afirmar que la mayoría de los adolescentes evaluados han sido expuesto involuntariamente a material sexual online, dato que llama especialmente la atención si tenemos en cuenta que los principales estudios sobre El estiman una prevalencia de entre 23-44\% (Jones et al., 2012; Mitchell et al., 2003; Mitchell et al., 2007). Estas diferencias en la prevalencia pueden deberse a factores políticos, sociales y/o culturales dado que los mencionados estudios se han llevado a cabo en otro contexto, concretamente en EE.UU. donde el acceso a contenido ilegal a través de internet parece ser más restrictivo y estar sometido a una regulación más punitiva que la que podamos encontrar en Europa. De hecho, algunos estudios realizados en Europa (Buljan-Flander, Cosic \& Profaca, 2009) o países donde la regulación a nivel legal es similar o incluso menos restrictiva que la nuestra, como Australia (Flood, 2007; Flood \& Hamilton, 2003), muestran porcentajes de El bastante parecidos al obtenido en nuestro estudio. Asimismo, y siendo consistentes con la literatura existente sobre el tema, nuestros hallazgos revelan que los chicos se han expuesto más que las chicas de forma involuntaria a pornografía en internet (Chang et al., 2016; Sabina, Wolak \& Finkelhor, 2008; Ybarra \& Mitchell, 2005) y también lo han hecho a una edad más temprana (Sabina et al., 2008).

Otro aspecto relevante sobre la El aunque muy poco estudiado en la literatura revisada es el tipo de contenido al que nuestros adolescentes han sido expuestos. En este sentido y a partir de los datos obtenidos podemos afirmar que las escenas más vistas han sido gente desnuda que no está 
teniendo relaciones sexuales, relaciones sexuales entre hombre-mujer, y personas desnudas enseñando los genitales. Aunque en menor medida también es necesario tener en consideración que dentro del contenido visto por los adolescentes aparecen escenas de sometimiento, violencia sexual, y actividad sexual con niños pequeños por citar algunos contenidos que estarían dentro de la ilegalidad. Estos mismos contenidos también han sido encontrados en un estudio previo con porcentajes incluso mayores (Sabina et al., 2008).

Finalmente, y teniendo en cuenta que la literatura habla de efectos negativos de la El (Owens et al., 2012; Ševíková et al., 2016) en nuestro estudio hemos intentado profundizar en el análisis del impacto de la El encontrando entre las principales consecuencias tras la exposición, los síntomas de evitación/embotamiento emocional, síntomas sexuales y aumento de activación fisiológica. En menor medida, los adolescentes también presentan síntomas de reexperimentación del suceso. Cabe señalar que estas consecuencias varían en función del género, de la edad de la primera exposición y también del tipo de contenido visto. En función del género las diferencias muestran que los síntomas explorados son todos más frecuentes en chicos que en chicas, excepto los relativos al grupo de síntomas de evitación. En cuanto a la edad de la primera exposición también podemos constatar que aparecen correlaciones con síntomas de cada uno de los bloques analizados indicando mayor presencia de síntomas cuanto más pronto fueron expuestos los adolescentes de forma involuntaria a contenido sexual online. De igual forma el tipo de escena visto también influye en las consecuencias experimentadas. En este sentido destacarían ciertos contenidos, como por ejemplo el ver escenas que implican sometimiento, actividad sexual con adolescentes y actividad sexual con niños pequeños por ser los contenidos que más diferencias han ofrecido.

Este estudio ofrece datos relevantes a tener en consideración sobre todo de cara a implementar programas de formación afectivo-sexual y de prevención que minimicen el impacto y las consecuencias negativas derivadas de una El a material sexual online y además a edades tempranas dadas las implicaciones que ello podría tener en el desarrollo psicosexual. No obstante, es necesario seguir profundizando en su estudio ampliando datos de más zonas de nuestra geografía que nos permitan confirmar y generalizar los hallazgos obtenidos.

\section{REFERENCIAS BIBLIOGRÁFICAS}

Ballester-Arnal, R., Giménez-García, C., Gil-Llario, M.D. \& Castro-Calvo, J. (2016). Cybersex in the "Net generation": Online sexual activities among Spanish adolescents. Computers in Human Behavior, 57, 261-266.

Bryant, C. (2009). Adolescence, pornography and harm. Trends \& Issues in Crime and Criminal Justice, 368, 1-6.

Buljan-Flander, G., Cosic, I. \& Profaca, B. (2009). Exposure of children to sexual content on the Internet in Croatia. Child Abuse \& Neglect, 33, 849-856.

Chang, F.C., Chiu, C.H., Miao, N.F., Chen, P.H., Lee, C.M. \& Chiang, J.T. (2016). Predictors of unwanted exposure to online pornography and online sexual solicitation of youth. Journal of Health Psychology, 21(6), 1107-1118.

Delmonico, D., \& Griffin, E. (2008). Cybersex and the e-teen: what marriage and family therapists should know. Journal of Marital and Family Therapy, 34, 431-444.

Eurostat (2015). Being young in Europe today. Eurostat Statistical Books. Luxembourg: Publications Office of the European Union. Disponible: http://ec.europa.eu/eurostat/documents/3217494/6776245/KS-05-14-031-EN-N.pdf/18bee6f0-c181-457d-ba82-d77b314456b9.

Flood, M. (2007). Exposure to pornography among youth in Australia. Journal of Sociology, 43 (1), 45-60. 


\section{CONSECUENCIAS DE LA EXPOSICIÓN INVOLUNTARIA A MATERIAL SEXUAL EN ADOLESCENTES}

Flood, M. \& Hamilton, C. (2003). Youth and pornography in Australia: evidence on the extent of exposure and likely effects (informe de The Australia Institute №52). Canberra: The Australia Institute.

Jones, L. M., Mitchell, K. y Finkelhor, D. (2012). Trends in Youth Internet Victimization: Findings From Three Youth Internet Safety Surveys 2000-2010. Journal of Adolescent Health, 50 (2), 179-186.

Instituto Nacional de estadística, INE. (2017). Encuesta sobre Equipamiento y Uso de Tecnologías de la Información y Comunicación en los hogares 2017. Consultado el 29 de marzo de 2017 en http://www.ine.es/dyngs/INEbase/es/operacion.htm?c=Estadistica_C\&cid=1254736176741\&m enu=ultiDatos\&idp $=1254735976608$

Livingstone, S., Haddon, L., Görzig, A., \& Ólafsson, K. (2011). Risks and safety on the internet: The perspective of European children. Full Findings. LSE, London: EU Kids Online.

Mitchell, K. J., Finkelhor, D. \& Wollak, J. (2003). The exposure of youth to unwanted sexual material on the Internet: A national survey of risk, impact, and prevention. Youth \& Society, 34, 330358.

Mitchell, K. J., Wolak, J. \& Finkelhor, D. (2007). Trends in youth reports of sexual solicitations, harassment and unwanted exposure to pornography on the Internet. Journal of Adolescent Health, 40, 116-126.

Observatorio de la Infancia en Andalucía (2010). Encuesta sobre equipamientos y uso de tecnologías de la información y la comunicación en los hogares 2009. Consejería de Innovación, Ciencia y Empresa. Junta de Andalucía.

Owens, E. W., Behun, R. J, Manning, J. C. \& Reid, R. C. (2012). The Impact of Internet Pornography on Adolescents: A Review of the Research. Sexual Addiction \& Compulsivity, 19, 99-122.

Peter, J. \& Valkenburg, P.M. (2008). Adolescents' exposure to sexually explicit Internet material and sexual preoccupancy: A three-wave panel study. Media Psychology 11(2), 207-234.

Sabina, C., Wolak, J. \& Finkelhor, D. (2008) The nature and dynamics of internet pornography exposure for youth. CyberPsychology \& Behavior, 11(6), 1-3.

Ševíková, A., Simon, L., Daneback, K. \& Kvapilík, T. (2016). Bothersome Exposure to Online Sexual Content Among Adolescent Girls. Youth \& Society, 47(4) 486-501.

Wollack, J., Mitchell, K. y Finkelhor, D. (2007). Unwanted and Wanted Exposure to Online Pornography in a National Sample of Youth Internet Users. Pediatrics, 119 (2), 247-257.

Ybarra, M.L. \& Mitchell, K.J. (2005) Exposure to internet pornography among children and adolescents: A national survey. CyberPsychology \& Behavior, 8(5), 473-486. 